\title{
Asma metabólica: evidências atuais e perspectivas terapêuticas
}

\author{
Mara M. R. Félix, ${ }^{1}$ Érica A. O. C. Jordão, ${ }^{1}$ Fábio C. Kuschnir ${ }^{2, *}$
}

\begin{abstract}
Resumo
Asma e obesidade estão associadas tanto do ponto de vista epidemiológico como fisiopatológico. Estudos longitudinais indicam que, de modo geral, a obesidade precede a asma e que o risco relativo de asma aumenta com a obesidade. Além disso, a redução de peso em obesos asmáticos resulta em declínio da gravidade da doença. O chamado "fenótipo obeso" da asma na infância apresenta características diferenciadas de função pulmonar, hiperreatividade brônquica, além de inflamação não eosinofílica das vias aéreas e um pior controle dos sintomas com corticosteroides inalatórios. Mais recentemente, tem sido observada também a associação entre asma e síndrome metabólica (SM) e seus componentes como a resistência insulínica, hiperglicemia e a hipertensão arterial, independentemente do índice de massa corporal. Nestes casos, a disfunção oxidativa do epitélio brônquico e a inflamação sistêmica relacionada à SM parecem contribuir para a patogênese da asma. Neste artigo de revisão, são discutidas as evidências científicas atuais acerca dos mecanismos fisiopatológicos da associação entre a asma e os componentes da SM, além de perspectivas futuras no manejo dos pacientes com essas doenças crônicas não transmissíveis de alta prevalência e morbidade na infância e adolescência.
\end{abstract}

Descritores: Asma; Obesidade; Síndrome metabólica; Infância.

\section{Abstract \\ Metabolic asthma: Current evidence and therapeu- tic perspectives}

Asthma and obesity are associated from both the epidemiological and pathophysiological point of view. Longitudinal studies indicate that, in general, obesity precedes asthma, and that relative risk for asthma increases with obesity. Furthermore, weight reduction in obese asthmatics results in decreased severity of the disease. The so-called "obese phenotype" of childhood asthma has distinct characteristics of lung function, bronchial hyperresponsiveness, non-eosinophilic airway inflammation and worse control of symptoms with inhaled corticosteroids. More recently, it has also been observed the association between asthma and metabolic syndrome (MS) and its components such as insulin resistance, hyperglycemia and hypertension, regardless of body mass index. Oxidative dysfunction of the bronchial epithelium and systemic inflammation associated with MS appear to contribute to the pathogenesis of asthma. This review article discusses the current scientific evidence about the pathophysiological mechanisms of the association between asthma and components of the metabolic syndrome, as well as future prospects in the management of patients with these chronic diseases of high prevalence and morbidity in childhood and adolescence.
1. Programa de Pós-Graduação em Ciências Médicas. Faculdade de Ciências Médicas. Universidade do Estado do Rio de Janeiro. Rio de Janeiro, RJ, Brasil.

2. Departamento de Pediatria. Faculdade de Ciências Médicas. Universidade do Estado do Rio de Janeiro. Rio de Janeiro, RJ, Brasil.

*Endereço para correspondência:

HUPE, Departamento de Pediatria

Boulevard Vinte e Oito de Setembro, 77, 2ㅇ andar.

Rio de Janeiro, RJ, Brasil. CEP 20551-030

E-mail: fabkuschnir@gmail.com

Revista HUPE, Rio de Janeiro, 2016;15(2):163-169

doi: 10.12957/rhupe.2016.28242

Recebido em 13/06/2016. Aprovado em 23/09/2016.

Keywords: Asthma; Obesity; Metabolic syndrome; Childhood.

\section{Resumen}

\section{Asma metabólico: La evidencia actual y perspecti- vas terapéuticas}

El asma y la obesidad están asociados tanto desde el punto de vista fisiopatológico como epidemiológico. Los estudios longitudinales indican que, en general, la obesidad precede al asma y que el riesgo relativo de asma aumenta con la obesidad. Además, la reducción de peso en pacientes obesos con asma redujo la gravedad de la enfermedad. El llamado "fenotipo obeso" del asma infantil tiene características distintas de la función pulmonar, hiperreactividad bronquial, inflamación no eosinofílica de las vías respiratorias y peor control de los síntomas con corticoesteroides inhalados. Recientemente, se ha observado también la asociación entre el asma y el síndrome metabólico (SM) y sus componentes, como resistencia a la insulina, hiperglucemia e hipertensión, independientemente del índice de masa corporal. En estos casos, la disfunción oxidativa del epitelio bronquial y la inflamación sistémica asociada con el SM parecen contribuir a la patogénesis del asma. Este artículo discute la evidencia científica actual sobre los posibles mecanismos fisiopatológicos de la asociación entre el asma y los componentes del SM, así como las perspectivas de futuro en el tratamiento de los pacientes con estas enfermedades crónicas de alta prevalencia y morbilidad en la niñez y la adolescencia.

Palabras clave: Asma; Obesidad; Síndrome metabólico; Niñez. 


\section{Artigo de revisão}

\section{Introdução}

Asma é uma doença crônica inflamatória das vias aéreas caracterizada pela presença de hiperresponsividade brônquica e obstrução variável do fluxo aéreo pulmonar, levando a episódios recorrentes de sibilos, dispneia e tosse. Constitui problema mundial de saúde pública, atingindo todas as faixas etárias, em especial crianças e adolescentes. ${ }^{1}$

Segundo dados do International Study of Asthma and Allergies in Childhood (ISAAC), ${ }^{2}$ estudo multicêntrico mundial criado para estimar a prevalência de asma, rinoconjuntivite alérgica e eczema atópico em crianças de 6 e 7 anos e adolescentes de 13 e 14 anos, a prevalência média global de asma ativa nestas faixas etárias foram respectivamente de 11,1\% e 13,7\%. No Brasil, estes índices foram de $24,3 \%$ e $19 \%$, respectivamente. Trata-se de um dos mais elevados entre os países da América Latina, acarretando importante carga sobre o sistema de saúde. ${ }^{2}$ Mais recentemente, o Estudo de Riscos Cardiovasculares em Adolescentes (ERICA), envolvendo cerca de 75.000 adolescentes brasileiros de 12 a 17 anos, mostrou uma prevalência média de asma ativa de $13,4 \%{ }^{3}$

Asma e obesidade estão relacionadas do ponto de vista epidemiológico e fisiopatológico. Estudos longitudinais indicam que a obesidade precede a asma e que o risco relativo de asma aumenta com a obesidade. ${ }^{4}$ Além disso, a redução de peso em pacientes obesos asmáticos resulta em declínio da gravidade da asma. ${ }^{5}$

Mais recentemente, tem sido observada também a associação entre asma e síndrome metabólica (SM) e seus componentes como a resistência insulínica e a hipertensão arterial, independentemente do índice de massa corporal (IMC). ${ }^{6}$

A SM em adultos consiste na combinação de três ou mais dos seguintes critérios: deposição central de gordura, aumento de triglicerídeos, níveis baixos de HDL colesterol, hipertensão arterial sistêmica e glicemia de jejum elevada. Até o momento, não há uma definição amplamente aceita para crianças e adolescentes.? Critérios propostos pela International Diabetes Federation ${ }^{8}$ para esta faixa etária incluem: adiposidade (definida pela circunferência abdominal ou índice de massa corpórea - IMC); metabolismo glicêmico (definido pela glicemia de jejum elevada ou teste de tolerância à glicose oral alterado); dislipidemia (aumento de triglicerídeos ou níveis baixos de HDL colesterol) e hipertensão arterial (Tabela 1). Estudos avaliando a prevalência de síndrome metabólica em adolescentes apresentam ta- xas elevadas que variam de 2,2\% a 52,1\%, dependendo do local e metodologia utilizados. ${ }^{9}$ No Brasil, o ERICA estimou esta prevalência em $2,6 \% .^{10}$

A obesidade em crianças e adolescentes mostrou ser um importante preditor para a hipertensão arterial, elevação do colesterol total e de lipoproteínas séricas. ${ }^{11}$ Desse modo, crianças e adolescentes obesos já estão mais sujeitos ao desenvolvimento das diversas complicações clínico-metabólicas encontradas em adultos obesos. ${ }^{9}$

Assim como a asma, as prevalências de obesidade e de outros componentes da síndrome metabólica vêm aumentando progressivamente na infância e adolescência nas últimas décadas. ${ }^{12}$ Segundo os dados da Pesquisa de Orçamentos Familiares, realizada entre 2008/2009 pelo Instituto Brasileiro de Geografia e Estatística ${ }^{13}$, a prevalência de excesso de peso em adolescentes passou de 3,7\% em 1970, para 21,7\% em 2009.

Neste artigo de revisão, são abordados aspectos relacionados à associação entre a asma e os componentes da síndrome metabólica. Serão discutidas as principais evidências científicas acerca dos mecanismos fisiopatológicos dessa associação, além de perspectivas futuras de manejo dessas doenças crônicas não transmissíveis (DCNT) de alta prevalência e morbidade na infância e adolescência.

\section{Mecanismos fisiopatológicos}

Asma e obesidade

\section{Tabela 1. Diagnóstico de síndrome metabólica.}

Presença de pelo menos três dos componentes abaixo, com presença obrigatória de circunferência abdominal (CC) elevada.*

\section{$\mathrm{CC}$}

$<16$ anos: $\geq$ Percentil 90

$\geq 16$ anos, sexo masculino: $\geq 90 \mathrm{~cm}$

$\geq 16$ anos, sexo feminino: $\geq 80 \mathrm{~cm}$

HDL-C

$<16$ anos: $40 \mathrm{mg} / \mathrm{dL}$

$\geq 16$ anos, sexo masculino: $<40 \mathrm{mg} / \mathrm{dL}$

$\geq 16$ anos, sexo feminino: $<50 \mathrm{mg} / \mathrm{dL}$

\section{Triglicerídeos}

$\geq 150 \mathrm{mg} / \mathrm{dL}$

Glicose

$\geq 100 \mathrm{mg} / \mathrm{dL}$

Pressão arterial sistólica

$\geq 130 \mathrm{mmHg}$ ou diastólica $\geq 85 \mathrm{mmHg}$

* De acordo com os critérios da International Diabetes Federation - IDF. ${ }^{8}$ 
A asma é uma doença heterogênea com diferentes fenótipos e endótipos. ${ }^{1}$ Os fenótipos da asma na infância representam diferentes padrões de características clínicas dependentes de diversos fatores, entre os quais a época de início da doença, presença de atopia e resposta terapêutica aos corticosteroides inalatórios. Os endótipos de asma se referem a subtipos de asma definidos por padrões moleculares ou de mecanismos subjacentes de desenvolvimento da doença. ${ }^{14}$

Embora o espectro clínico da asma seja bem amplo, a presença de inflamação nas vias aéreas é uma característica comum a todas as formas, desde as mais leves até aquelas mais graves e persistentes. ${ }^{1}$ Este processo envolve a participação de mastócitos, eosinófilos, linfócitos T CD4+ e de seus mediadores, além da produção de interleucinas (IL) responsáveis pela manutenção do estado inflamatório. ${ }^{15}$

De um modo geral, a asma infanto-juvenil apresenta um padrão predominante de linfócitos do tipo T-helper 2 (Th2), cujo repertório de citocinas compreende IL-4, IL-5 e IL- 13 e infiltrado inflamatório eosinofílico. Entretanto, nas formas mais graves e crônicas, de início mais tardio, pode prevalecer um padrão Th1 que secreta principalmente interferon- $\gamma$ (INF- $\gamma$ ) e fator de necrose tumoral-alfa (TNF-alfa), com predomínio de neutrófilos nas vias aéreas. ${ }^{15,16}$

A asma em obesos pode constituir um dos muitos fenótipos atribuídos à doença. Um subgrupo do chamado "fenótipo obeso" da asma, composto por crianças não atópicas com ganho de peso na infância precoce, apresenta características diferenciadas de função pulmonar, hiperreatividade brônquica, inflamação não eosinofílica das vias aéreas e pobre controle dos sintomas mesmo com a utilização de corticosteroides inalatórios. Entretanto, um grande número de crianças obesas asmáticas é formado por alérgicos com predomínio de padrão Th2 e que desenvolveram obesidade posteriormente. ${ }^{14}$

A relação entre asma e obesidade envolve fatores genéticos, fisiológicos e ambientais. Não há um mecanismo único que seja dominante na relação causal entre estas doenças, e vários fatores podem atuar em diferentes estágios do desenvolvimento do indivíduo, desde o período pré-natal até a idade adulta. ${ }^{17}$

Tantisira e Weiss ${ }^{16}$ ressaltam que a asma e a obesidade compartilham determinantes genéticos comuns, como o gene para o receptor $\beta$-adrenérgico localizado no cromossomo 5q, o gene para o TNF-alfa 33, o receptor beta para glicocorticoide e o gene da leptina.

Existem evidências de que a nutrição da gestante possa influenciar na evolução para o fenótipo de asma associado à obesidade através de alterações epigenéticas, que são modificações do genoma herdadas pelas próximas gerações, mas que não alteram a sequência do DNA. Além disso, o peso ao nascimento, tanto baixo como alto, aumenta o risco de desenvolvimento de sobrepeso e asma. ${ }^{17}$ Em particular, o baixo peso ao nascimento é um reconhecido fator de risco independente para a associação asma-obesidade. ${ }^{18}$ Já o aleitamento materno tem sido apontado como um fator protetor tanto para a asma quanto para a obesidade. ${ }^{19,20}$

A obesidade é considerada um estado pró-inflamatório, onde se observa o aumento dos níveis plasmáticos de proteína $\mathrm{C}$ reativa (PCR) e citocinas inflamatórias como TNF-alfa e IL-6, além de moléculas de adesão. Diferentes mecanismos sugerem que a ação pró-inflamatória do tecido adiposo pode levar ao desenvolvimento de inflamação nas vias aéreas e como consequência deste processo, à asma. ${ }^{16}$

\section{Asma e síndrome metabólica}

A SM é associada com a alteração de uma variedade de tipos celulares, incluindo as células endoteliais (CE), células epiteliais renais e epitélio glandular, e especula-se que as células do epitélio de vias aéreas também estejam alteradas. ${ }^{21} \mathrm{O}$ endotélio intacto libera localmente óxido nítrico (NO) através da NO-sintetase-endotelial (eNOS), regulada pela L-arginina. Esta substância em pequenas quantidades induz à vasodilatação local, sendo conhecida como fator de relaxamento dependente do endotélio. Entretanto, a função do NO no sistema respiratório é paradoxal, uma vez que em altas concentrações pode causar dano ao epitélio e edema na mucosa. ${ }^{22}$

As CE participam da resposta imune, expressando Toll-like receptors que, quando ativados, induzem à expressão de moléculas de adesão, NO-sintetase-induzível-2 (iNOS-2), endotelina, IL-1, além de outras moléculas com ações inflamatórias. A iNOS-2 aumenta a síntese de NO local que, na presença de oxigênio produz compostos nitrogenados reativos, levando a estresse oxo-nitrosativo e causando modificação e inibição de diversas proteínas importantes para a função celular. O pulmão do paciente asmático pode apresentar um estado de maior catabolismo da L-arginina com depleção da eNOS. ${ }^{22,23}$

Os hormônios também influenciam a relação entre a asma e a obesidade. ${ }^{17} \mathrm{O}$ tecido adiposo é atualmente considerado um órgão endócrino ativo que secreta vários mediadores, conhecidos como adipocinas. Entre 
os quais a leptina e a adiponectina. ${ }^{24}$

A leptina estimula a resposta Th1 e leva à produção de outras citocinas inflamatórias Em condições normais, ela age no hipotálamo como um supressor de apetite, entretanto em obesos parece ocorrer uma resistência à leptina. ${ }^{25}$ Estudos transversais demonstram aumento nos níveis circulantes de leptina em crianças obesas comparadas às não obesas. ${ }^{24,25}$ Além disso, os níveis de leptina também estão elevados em crianças asmáticas com peso normal quando comparadas às não asmáticas. A leptina também pode contribuir para a patogênese da asma por meio do remodelamento da via aérea induzido pelo vascular endothelial growth factor (VEGF), uma vez que esta adipocina estimula a liberação do VEGF pelas células do músculo liso das vias aéreas. ${ }^{25}$

A adiponectina é um mediador anti-inflamatório, que inibe o fator nuclear kappa-B (NF-kB), a IL-6 e o TNF-alfa, também aumenta a produção de IL-10 e do antagonista do receptor de IL-1. Ela é responsável pelo aumento da sensibilidade à insulina. Níveis reduzidos de adiponectina são encontrados em crianças e adolescentes com obesidade e estão associados a níveis elevados de PCR. ${ }^{24,25}$

A figura 1 demonstra as possíveis conexões genéticas, ambientais e fisiopatológicas entre a asma e a síndrome metabólica.

\section{Evidências epidemiológicas da conexão entre a asma e os componentes da síndrome metabólica}

Vários estudos epidemiológicos demonstram a associação entre a asma e a obesidade. ${ }^{14}$ Estudos longitudinais mostram que a obesidade precede a asma e aumenta o risco de incidência desta última. ${ }^{26} \mathrm{Em}$ crianças pequenas, o aumento rápido de peso pode levar a uma desregulação do desenvolvimento das vias aéreas e sibilância. Além disso, a obesidade materna e o ganho de peso durante a gestação podem aumentar a incidência de sibilância na infância..$^{27,28}$

A causalidade entre a asma e a obesidade pode ser bidirecional. Estudos já demonstraram um maior aumento de peso em asmáticos comparados a não asmáticos e fatores que favorecem o ganho de peso, como a redução da atividade física e o tratamento com corticosteroides orais podem estar presentes em crianças asmáticas..$^{29,30,14}$ Entretanto, a redução da atividade física em crianças asmáticas não é universal, podendo constituir um fator de confusão na associação entre asma e obesidade.

Mais recentemente a asma também tem sido associada à resistência insulínica e ao aumento dos triglicerídeos independentemente do IMC. ${ }^{31,32}$ Cottrell

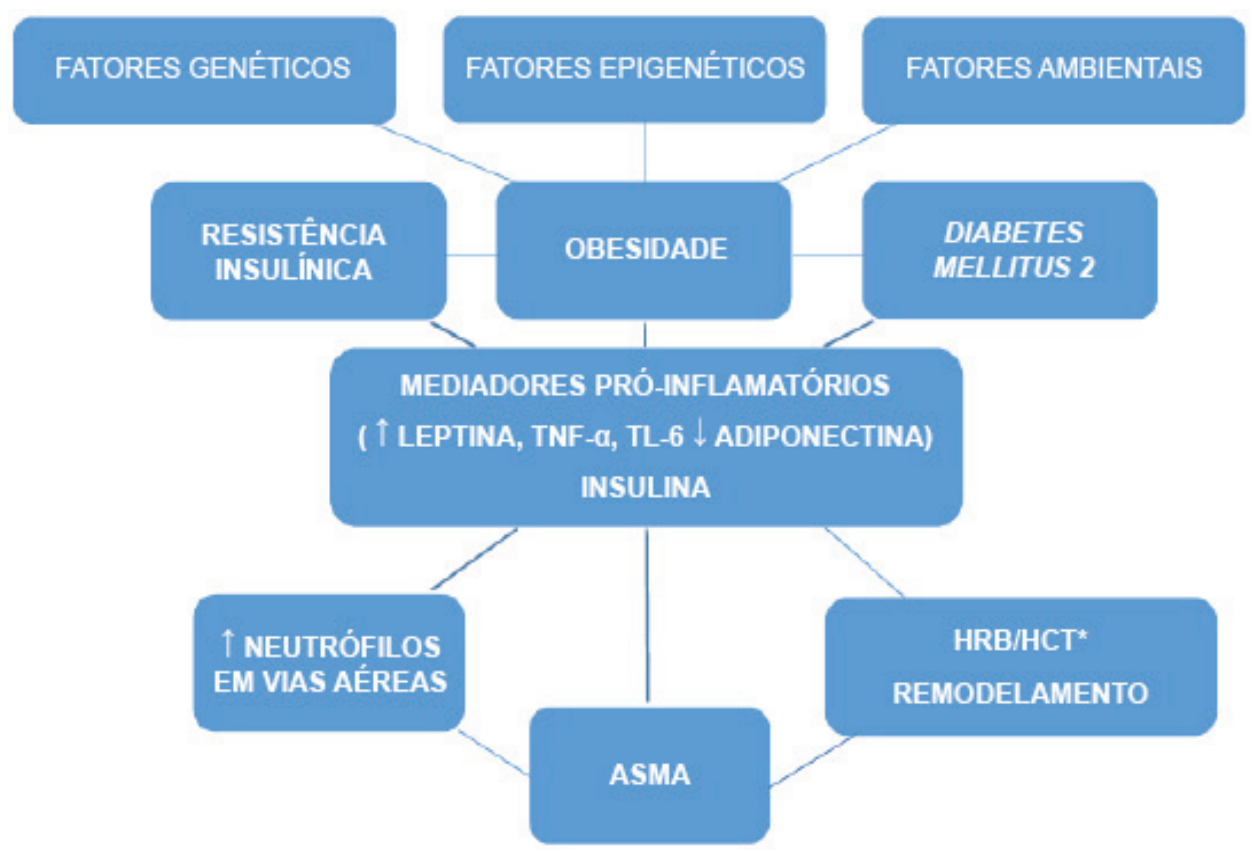

Figura 1. A obesidade, a resistência insulínica e o diabetes mellitus 2 estão relacionados à inflamação sistêmica e das vias aéreas e ao remodelamento, contribuindo para a patogênese da asma.

*HRB: hiperresponsividade brônquica; HCT: hipercontratilidade brônquica 
e colaboradores ${ }^{32}$ evidenciaram em estudo transversal que crianças asmáticas apresentavam maiores níveis de triglicerídeos e acantose nigricans que as não asmáticas. Assim, a hiperinsulinemia e a dislipidemia, que são precursores silenciosos do diabetes e da doença cardiovascular, poderiam estar associadas ao desenvolvimento da asma, além de confundir a relação epidemiológica entre esta última e a obesidade. ${ }^{31}$

Mais recentemente, Forno e colaboradores ${ }^{33}$ investigaram a associação entre obesidade, sensibilidade insulínica, síndrome metabólica e função pulmonar em pacientes com e sem asma em adolescentes americanos. Foi observado que a resistência insulínica associou-se negativamente ao volume expiratório forçado no $1^{\mathrm{o}}$ segundo $\left(V_{E F}{ }^{1}\right)$ e capacidade vital forçada (CVF) em adolescentes com e sem asma, enquanto a SM foi associada à menor relação $\mathrm{VEF}^{1} / \mathrm{CVF}$, com redução mais pronunciada entre pacientes asmáticos. Observou-se ainda que o IMC mais elevado esteve associado à redução da relação $\mathrm{VEF}^{1} / \mathrm{CVF}$ em adolescentes com resistência insulínica. Quando comparados aos adolescentes saudáveis, os adolescentes com SM tiveram uma redução de $2 \%$ na relação $\mathrm{VEF}^{1} / \mathrm{CVF}$, entre os asmáticos esta redução foi de $6 \%$, enquanto que naqueles com SM e asma concomitante houve uma queda de $10 \%$ nessa relação que foi significativamente estatística $(\mathrm{p}<0,05)$. Os autores concluíram que a SM, a obesidade e a resistência insulínica podem reduzir a função pulmonar independentemente da presença de asma. ${ }^{33}$

Além da avaliação da resistência insulínica dentro do contexto da obesidade, há uma preocupação atual em relação aos pacientes com anormalidades metabólicas, sem alteração de peso corporal, o chamado fenótipo obeso-magro (thin-fat) Estes adolescentes estão predispostos às mesmas comorbidades cardiovasculares e respiratórias que os obesos, mas podem ser negligenciados por serem eutróficos. ${ }^{31}$

\section{Perspectivas futuras}

De modo geral, a asma é associada aos componentes da síndrome metabólica de forma independente, especialmente obesidade, níveis elevados de triglicerídeos e diabetes mellitus. ${ }^{31,32}$ Contudo, os mecanismos fisiopatológicos pelos quais essas entidades se associam ainda não foram completamente elucidados, não permitindo, até o momento, definir de modo pleno a relação de causalidade entre elas. Levando em consideração que a asma é uma doença multifatorial com diversos fenótipos, é possível especular que a SM e seus componentes possa influenciar a asma de forma diferente conforme o fenótipo envolvido. Alguns estudos têm apontado futuras e promissoras abordagens terapêuticas para estes pacientes.

Dentre os componentes da síndrome metabólica, a obesidade é o de maior evidência de associação com a asma, inclusive com estudos demonstrando uma relação de causalidade entre estas condições. ${ }^{34}$ Apesar de não termos clareza de como a obesidade influencia cada fenótipo da asma, a principal diretriz internacional sobre a doença em sua versão mais recente já recomenda a perda de peso para os pacientes obesos com um grau B de evidência científica. ${ }^{1}$

A via da L-arginina-NO, extensamente estudada nas doenças cardiovasculares, é um mecanismo possível na gênese da asma metabólica e com grande potencial terapêutico para o futuro. Como citado anteriormente, o papel do NO nas vias aéreas depende de seu nível local: em concentração elevada leva a um estado de estresse oxidativo em vias aéreas com dano tecidual, enquanto que em níveis baixos tem um efeito broncodilatador. Supõe-se que a suplementação de L-arginina possa ser benéfica na asma, pois ao aumentar o substrato para a NOS, ocorre diminuição da produção de radicais livres, diminuindo o estresse oxidativo. ${ }^{35}$

As estatinas também já foram estudadas para esse fim, uma vez que ao inibirem a hidroximetilglutaril-CoA, diminuem o colesterol e a disponibilidade de isoprenoide necessário para a produção de GTPases que participam da sinalização celular. A disponibilidade reduzida dessas moléculas prejudica a transdução dos sinais das citocinas e quimiocinas, diminuindo a inflamação e hipertrofia celular. ${ }^{35}$ É importante ressaltar que poucos estudos clínicos duplo-cegos randomizados avaliando os efeitos das estatinas foram realizados em asmáticos até o momento, com resultados por vezes conflitantes, havendo necessidade de mais estudos nesta área de pesquisa a fim de observarmos melhor o efeito destes medicamentos sobre a asma. ${ }^{36}$

A resistência insulínica é outro componente da síndrome metabólica com reconhecida associação com a asma. ${ }^{32}$ Apesar de os efeitos da insulina e hiperglicemia serem bem conhecidos no diabetes mellitus, ainda não está bem estabelecido como ela atua em nível pulmonar. ${ }^{21}$

A insulina parece ter efeito anti-inflamatório em um ambiente Th1, porém parece induzir linfócitos T ainda não diferenciados (naive) à resposta do tipo Th2 . Além disso, é associada ao aumento da contratilidade do músculo liso, proliferação celular e fibrose nas vias aéreas inferiores. ${ }^{37}$ Coletivamente, estes achados 


\section{Artigo de revisão}

sugerem que a metformina tem potencial para atuar como adjuvante na terapia da asma. Inicialmente, um dos efeitos da metformina é a perda de peso corporal, que por si só já pode beneficiar o paciente asmático obeso. ${ }^{35}$ Além disso, essa substância aumenta a expressão e atividade de AMP-activated kinase (AMPK) em células do epitélio brônquico, diminuindo a secreção de citocinas pró-inflamatórias e reduzindo a inflamação. ${ }^{38}$ Entretanto, existem poucos estudos avaliando sua ação sobre a asma, e estes foram realizados apenas em modelos animais com resultados conflitantes. Deste modo, são necessárias mais pesquisas para uma melhor compreensão dos potenciais efeitos da metformina no tratamento de asmáticos com resistência insulínica.

O papel da alimentação na fisiopatogenia da síndrome metabólica é amplamente estudado. Sabe-se que o excesso de nutrientes sobrecarrega a mitocôndria, levando ao aumento da produção de espécies reativas de oxigênio que causam dano a esta organela celular. Assim, o processo de metabolização dos ácidos graxos para obtenção de energia é prejudicado com consequente aumento dos lipídios circulantes e hiperinsulinemia como uma resposta compensatória. Já o impacto deste aspecto específico da disfunção mitocondrial nas doenças respiratórias é menos conhecido. ${ }^{35}$

Levando em consideração essa possível via, propostas terapêuticas têm surgido, como a utilização de vitaminas com propriedades antioxidantes. Em 2009, Allen e colaboradores ${ }^{39}$ demonstraram que menores níveis de vitaminas A e $\mathrm{C}$ na dieta estavam associados com maior risco de asma, porém não encontraram a mesma associação com a vitamina E. Em recente meta-análise, realizada em 2014, foi observado que o consumo de frutas e vegetais crus foi significativamente associado a uma redução no risco de sibilos, provavelmente devido aos altos níveis de vitaminas e outros nutrientes antioxidantes nesses alimentos. ${ }^{40}$

\section{Conclusão}

A asma e a síndrome metabólica são DCNT complexas, onde múltiplos fatores parecem atuar sinergicamente para sua expressão. Mais estudos longitudinais de longo prazo são necessários para uma melhor compreensão dessa associação e seus mecanismos fisiopatológicos. A consolidação deste conhecimento permitirá o estabelecimento de novas estratégias preventivas e terapêuticas para auxiliar o crescente percentual de crianças e adolescentes que apresentam esse fenótipo da asma.

\section{Referências}

1. Global Initiative for Asthma (GINA). 2016 GINA Report: Global Strategy for Asthma Management and Prevention. Disponível em: http://ginasthma.org/2016-gina-report-global-strategy-for-asthma-management-and-prevention/ Acesso em: 10/04/2016.

2. Mallol J, Crane J, von Mutius E, et al. The International Study of Asthma and Alergies en Childhood (ISAAC) Phase Three: A global synthesis. Allergol Immunopathol. 2013;41(2):73-85. http://doi.org/10.1016/j.aller.2012.03.001

3. Kuschnir FC, Gurgel RQ, Solé D, et al. ERICA: prevalence of asthma in Brazilian adolescents. Rev Saude Publica. 2016;50(suppl 1):13s. http://doi.org/10.1590/S015188787.2016050006682

4. Guilliand FD, Berhane K, Islam T, et al. Obesity and the risk of newly diagnosed asthma in school-age children. Am J Epidemiol. 2003;158:406-15.

5. Stenius-Aarniala, Poussa T, Kvarnstrom J, et al. Immediate and long terms effects of weight reduction in obese people with asthma: randomized controlled study. BMJ. 2000;320:827-32.

6. Park J, Kim TB, Joo H, et al. Diseases concomitant with asthma in middle-aged and elderly subjects in Korea: a population-based study. Allergy, Asthma \& Immunol Res. 2013;5(1):16-25.

7. Pergher RNQ, Melo ME, Halpern A, et al. O diagnóstico de síndrome metabólica é aplicável às crianças? J. Pediatr. (Rio J.) [Internet]. 2010 Apr [cited 2016 Oct 19];86(2):101-108. Available from: http://www.scielo.br/scielo.php?script=sci_arttext\&pid=S0021-75572010000200004\&Ing=en. http://doi. org/10.2223/JPED.1983.

8. Zimmet P, Alberti G, Kaufman F, et al. The metabolic syndrome in children and adolescents. Lancet. 2007;369:2059-61.

9. Carneiro JR, Kuschnir MC, Clemente ELS, et al. Obesidade na adolescência: fator de risco para complicações clínico-metabólicas. Arq Bras Endocrinol Metab [Internet]. 2000 Oct [cited 2016 Oct 19];44(5):390-396. Available from: http://www.scielo.br/scielo.php?script=sci_arttext\&pid=S0004-27302000000500005\&Ing=en. http://doi. org/10.1590/S0004-27302000000500005.

10. Kuschnir MC, Bloch KV, Szklo M, et al. ERICA: prevalence of metabolic syndrome in Brazilian adolescents. Rev. Saúde Pública [Internet]. 2016 Feb [cited 2016 Oct 19];50(Suppl 1):11s. Available from: http://www.scielo.br/scielo.php?script=sci_arttext\&pid=S0034-89102016000200310\&Ing=en. Epub Feb 02, 2016. http://doi.org/10.1590/S01518-8787.2016050006701

11. National High Blood Pressure Education Program Working Group on High Blood Pressure in Children and Adolescents. The Fourth Report on Diagnosis, Evaluation, and Treatment of High Blood Pressure in Children and Adolescents. Pediatrics. 2004;114:555-76.

12. Kelishad R. Childhood overweight, obesity, and the metabolic syndrome in developing countries. Epidemiol Rev. 2007;29:62-76.

13. IBGE - Instituto Brasileiro de Geografia e Estatística, 2010. Pesquisa de Orçamentos Familiares (POF - 2008-2009): Antropometria e estado nutricional de crianças, adolescentes e adultos no Brasil. Disponível em: http://www.ibge.gov.br/home/ estatistica/populacao/condicaodevida/pof/2008_2009_encaa/ default.shtm. Acesso em: 15 de abril 2016

14. Lang JE. Obesity and Asthma in Children: Current and Future Therapeutic Options. Paediatr Drugs. 2014; 16(3): 179-188. http://dx.doi.org/10.1007/s40272-014-0069-1 
Mara M. R. Félix e col.. • Asma metabólica: evidências atuais e perspectivas terapêuticas

15. Holgate S T. Pathogenesis of asthma. Clin Exp Allergy 2008;38:872-897.

16. Tantisira KG, Weiss ST. Complex interactions in complex traits: obesity and asthma. Thorax. 2001;56 (Suppl II):64-74.

17. Frey U, Latzin P, Usemann J, et al. Asthma and obesity in children: current evidence and potential systems biology approaches. Allergy. 2015;70:26-40.

18. Mu M, Ye S, Bai MJ, et al. Birth weight and subsequent risk of asthma: a systematic review and meta-analysis. Heart Lung Circ. 2014;23(6):511-519. http://doi.org/10.1016/j. hlc.2013.11.018

19. Dogaru CM, Nyffenegger D, Pescatore AM, et al. Breastfeeding and childhood asthma: systematic review and meta-analysis. Am J Epidemiol. 2014;179:1153-1167.

20. Lefebvre CM, John RM. The effect of breastfeeding on childhood overweight and obesity: a systematic review of the literature. J Am Assoc Nurse Pract. 2013;26:386-401.

21. Agrawal A, Mabalirajan U, Ahmad T, et al. Emerging interface between metabolic syndrome and asthma. Am J Respir Mol Biol. 2011;44:270-275. http://doi.org/10.1165/rcmb.20100141TR

22. Brunini TMC, Perim N, Costa E, et al. O papel da via L-arginina-óxido nítrico nas doenças pulmonares. Pulmão RJ. 2006;15(3):184-190.

23. Hansson G, Libby P, Schönbeck U, et al. Innate and adaptative immunity in the pathogenesis of aterosclerosis. Circ Res. 2002;91:281-291.

24. Alikasifoglu A, Gönç EN, Özön ZA, et al. The relationship between serum adiponectin, tumor necrosis factor-alpha, leptina levels and insulin sensitivity in childhood and adolescent obesity: adiponectin is a marker of metabolic syndrome. J Clin Res Ped Endo. 2009;1(5):233-239.

25. Jensen ME, Colins CE, Gibson PG, et al. The obesity phenotype in children with asthma. Paediatric Respiratory Reviews. 2011;12:152-159. http://doi.org/10.1016/j.prrv.2011.01.009

26. Beuther DA, Sutherland ER. Overweight, obesity, and incident asthma: a meta-analysis of prospective epidemiologic studies. Am J Respir Crit Care Med. 2007;175(7):661-6.

27. Rzehak P, Wijga AH, Keil T, et al. Body mass index trajectory classes and incident asthma in childhood: results from $8 \mathrm{Eu}$ ropean Birth Cohorts--a Global Allergy and Asthma European Network initiative. J Allergy Clin Immunol. 2013;131(6):152836. http://doi.org/10.1016/j.jaci.2013.01.001

28. Pike KC, Inskip HM, Robinson SM, et al. The relationship between maternal adiposity and infant weight gain, and child- hood wheeze and atopy. Thorax. 2013;68:372-9. http://doi. org/10.1136/thoraxjnl-2012-202556

29. Jones SE, Merkle SL, Fulton JE, et al. Relationship between asthma, overweight, and physical activity among U.S. high school students. J Community Health. 2006;31(6):469-78.

30. Glazebrook C, McPherson AC, Macdonald IA, et al. Asthma as a barrier to children's physical activity: implications for body mass index and mental health. Pediatrics. 2006; 118(6):2443-9.

31. Perez MK, Piedimonte G. Metabolic asthma: Is there a link between obesity, diabetes, and asthma? Immunol Clin North Am. 2014;34:777-784. http://dx.doi.org/10.1016/j.iac.2014.07.002

32. Cottrell L, Neal WA, Ice C, et al. Metabolic abnormalities in children with asthma. Am J Respir Crit Care Med. 2011;183:441-8.

33. Forno E, Han YY, Muzumdar RH et al. Insulin resistance, metabolic syndrome, and lung function in US adolescents with and without asthma. J Allergy Clin Immunol. 2015;136:304-11.

34. Egan KB, Ettinger AS, Bracken MB. Childhood body mass index and subsequent physician-diagnosed asthma: a systematic review and meta-analysis of prospective cohort studies. BMC Pediatr. 2013;13:121. http://doi.org/10.1186/1471-243113-121

35. Linderholm AL, Bratt JM, Schuster GU, et al. Novel therapeutic strategies for adult obese asthmatics. Immunol Allergy Clin North Am. 2014;34(4):809-23. http://doi.org/10.1016/j. iac.2014.07.006

36. Zeki AA. Statins and asthma: where we stand, and the next critical steps in research. Curr Med Res Opin 2014;30(6):10511054. http://doi.org/10.1185/03007995.2014.881794

37. Singh S, Prakash YS, Linneberg A, et al. Insulin and the lung: connecting asthma and metabolic syndrome. J Allergy. 2013;13: Article ID 627384, 8 pages. http://doi. org/10.1155/2013/627384

38. Calixto MC, Lintomen L, André DM, et al. Metformin attenuates the exacerbation of the allergic eosinophilic inflamation in high fat-diet-induced obesity in mice. PLoS One. 2013;24;8(10):e76786. http://doi.org/10.1371/journal. pone.0076786

39. Allen S, Britton JR, Leonardi-Bee A. Association between antioxidant vitamins and asthma outcome measures: systematic review and meta-analysis. Thorax. 2009;64(7):610-9. http:// dx.doi.org/10.1136/thx.2008.101469

40. Seyedrezazadeh E, Moghaddam MP, Ansarin K, et al. Fruit and vegetable intake and risk of wheezing and asthma: a systematic review and meta-analysis. Nutr Rev. 2014;72(7):411-28. http://doi.org/10.1111/nure.12121. 